\title{
MiR-34a Enhances Chondrocyte Apoptosis, Senescence and Facilitates Development of Osteoarthritis by Targeting DLL1 and Regulating PI3K/AKT Pathway
}

\author{
Wei Zhang Peichun Hsu Biao Zhong Shang Guo Chi Zhang \\ Yukai Wang Congfeng Luo Yulin Zhan Changqing Zhang \\ Department of Orthopaedics, Shanghai Jiao Tong University Affiliated Sixth People's Hospital, \\ Shanghai, China
}

\section{Key Words}

Mir-34a • Apoptosis $・$ Senescence $\cdot$ OA $・$ DLL1 $・$ PI3K-AKT

\begin{abstract}
Background/Aims: Osteoarthritis (OA) is the prevalent degenerative disease caused by various factors. MicroRNAs are important regulators in the inflammation and immune response. The aim of this study was to investigate the effect of microRNA-34a (MiR-34a) on the death of chondrocytes, senescence, as well as its role in OA progression. Methods: A series of experiments involving CCK-8, flow cytometry, $\beta$-galactosidase staining and wound healing assays were conducted to determine the cellular capabilities of proliferation, cell apoptosis, senescence and the ability of cells to recover from injury, respectively. Binding sites between miR-34a and delta-like protein 1 (DLL1) were identified using a luciferase reporter system, whereas mRNA and protein expression of target genes was determined by RT-PCR and immunoblot, respectively. OA model was generated via surgery. Results: We found that miR-34a expression was increased in the cartilage of OA patients. In rat chondrocytes and chondrosarcoma cells, miR-34a transfections noticeably inhibited the expression of DLL1, triggered cell death and senescence, suppressed proliferation, and prevented scratch assay wound closure. However, transfection of a miR-34a inhibitor displayed adverse effects. Additionally, secretion and expression of factors associated with cartilage degeneration were altered via miR-34a. Moreover, miR-34a directly inhibits DLL1 mRNA. Furthermore, concentrations of DLL1, total PI3K, and $\mathrm{p}-\mathrm{AKT}$ declined in chondrocytes that overexpress miR34a. DLL1 overexpression elevated PI3K and p-AKT levels, and eliminated cell death triggered by a miR-34a mimic. In vivo, miR-34a remarkably inhibited miR-34a up-regulation, while enhanced the level of DLL1 expression. In the knee joints of surgery-induced OA rats, articular chondrocyte death and loss of cartilage were attenuated via miR-34a antagomir injection. Conclusions: These findings indicate that miR-34a contributes to chondrocyte death, causing OA progression through DLL1 and modulation of the PI3K/AKT pathway.

W. Zhang and P. Hsu contributed equally to this work.




\section{Introduction}

As a chronic degenerative joint disease, osteoarthritis $(\mathrm{OA})$ affects a variety of joints. Its features include the loss of cartilage, alterations of subchondral bone, synovitis, as well as other alterations of the joints [1,2]. OA manifests itself as pain, swelling, malformation, and decreased joint motion [3]. Risk factors for OA consist of age, obesity, genetics, abnormal anatomy, joint damage, and hypernomic weight bearing [4, 5]. Joint replacement serves as the dominant therapy to treat terminal OA [6]. The approaches to counteract and avoid OA remain insufficient. Significant evidence suggests that the death of chondrocytes participates in the incidence as well as the progression of OA [7, 8]. Various pathways and cytokines are involved in OA chondrocyte death, including nitrous oxide (NO), p38 MAPK, Fas, PI3K/AKT, TNF- $\alpha$, as well as IL-1 $\beta[9,10]$.

miRNAs are encoded by endogenic genes and participate in post-transcriptional modulation of gene expression in a variety of organisms, such as nematodes and men [11]. Regarding OA cartilage, evidence indicates that miRNA may participate in the death of chondrocytes [12,13]. Additionally, diverse miRNAs, such as miRNA-21, miRNA-448, and miRNA-142-3p $[13,14]$, participate in OA development. The group of human miR34 includes miR-34a, miR-34b, and miR-34c [15]. These family members serve as tumor inhibitors, which are crucial to the p53 pathway [16]. Previous research has revealed that the miR-34 family decreases proliferation, triggers cell death, and influences cell migration [17]. Replacement of miR-34 has been investigated in clinical trials in order to treat primary liver cancer as well as other kinds of malignancies with hepatic metastases [18]. Nevertheless, the understanding of the influence of miR-34 on OA is insufficient.

Notch signaling is a highly conserved juxtacrine-signaling pathway that is repeatedly used to coordinate the differentiation between adjacent cells [19]. The Notch ligand Dalalike 1 (DLL1) gene encodes one of the ligands in the Notch signaling pathway, which is highly conserved to regulate cell fate determination, proliferation, stem cell self-renewal and apoptosis [20]. Phosphatidylinositide 3 kinases (PI3K)/AKT signalling contribute to a variety of processes that are critical in mediating many aspects of cellular function, including nutrient uptake, anabolic reactions, cell growth, migration and survival [21]. Previously study showed that Notch2 depletion enhances the proliferative and invasive potential of cancer cells, via activation of PI3K/AKT pathway and ensuing increase in MMP9 activities [22]. Notch signaling regulates PI3K/AKT signaling pathway to participate in widely divergent cellular processes $[23,24]$.

In this study, we explored the influence of miR-34a on OA. Our research indicates that miR-34a promotes the death and senescence of chondrocytes. Additionally, miR-34a assists in OA generation via DLL1 through modulation of the PI3K/AKT pathway.

\section{Materials and Methods}

\section{Samples}

Knee joint and caput femoris cartilage were obtained from OA patients. Patients included two females and five males for hip OA (55 to 81 years old). Knee OA patients included three females and four males, whose ages ranged from 47 to 71 years. Cartilage tissues from patients (three females and five males, 43 to 72 years old) with collum femoris fractures who received total joint replacement served as controls. $\mathrm{OA}$ diagnosis was made according to medical history and $\mathrm{x}$-rays. The gross, pathologic observation was noted upon replacement of joint. Patients in the control group had healthy joints and cartilage. Our research obtained approval of the ethics committee of our hospital. Fully informed, written consent was obtained from every participant.

Separation and cultivation of chondrocytes

Primary chondrocytes were obtained from five neonatal Sprague-Dawley rats. In short, rats were euthanized under anesthesia. Cartilages in the rats of Articulatio genus were eliminated under sterile 


\section{Cellular Physiology Cell Physiol Biochem 2018;48:1304-1316

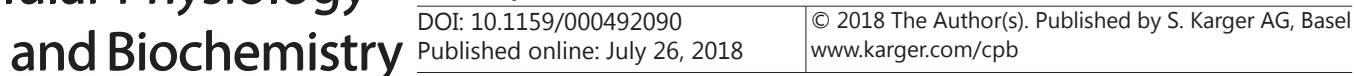 \\ Zhang et al.: MiR-34a Modulates Osteoarthritis}

conditions. Subsequent to the elimination of attaching muscle, connective tissue, as well as perichondrium, rest of the cartilages were minced and washed thrice with PBS. Tissues were digested by trypsin $(2.5 \mathrm{~g} / \mathrm{L})$ for fifteen minutes. Subsequent to supernatant elimination, PBS was utilized to wash the cartilages. Using a five to ten times volume of a type II collagenase solution ( $2 \mathrm{~g} / \mathrm{L})$, a four-hour digestion was carried out at $37^{\circ} \mathrm{C}$. The separated cells were purified using a nylon mesh sieve. Cells were centrifuged for eight minutes at $1000 \mathrm{r} / \mathrm{min}$. The proportion of apoptotic cells was evaluated. Separated chondrocytes were placed in cultivating flasks at $1 \times 10^{5}$ cells $/ \mathrm{ml}$. Cells were grown in incubator and the medium was renewed after forty-eight hours. Hs 819.T chondrosarcoma cells were obtained from ATCC and were cultivated in DMEM.

\section{Cells and cell culture}

Human chondrocytes (HC) derived from normal human articular cartilage were purchased from Cell Applications (San Diego, CA, USA). Cells were cultured in chondrocyte growth medium. Cells were maintained in a humidified incubator at $37{ }^{\circ} \mathrm{C}$ with $5 \% \mathrm{CO} 2$.

\section{Transient transfection for miR-34a functional assessment}

Injection of Hs 819.T cells and primary chondrocytes was carried out in six-well plates at $2.5 \times 10^{5}$ cells/well. Subsequent to the twenty-four-hour incubation, cells underwent six-hour transfections in DMEM without serum or antibiotics utilizing Lipofectamine 2000, miR-34a inhibitor, miR-34a mimic (Qiagen; Germantown, MD, USA), or controls at $30 \mathrm{nM}$ (Qiagen; Germantown, MD, USA). Following transfections, the media was replaced and cells were grown for forty-eight hours before additional manipulations.

Excessive expression of DLL1

Excessive DLL1 expression was acquired from Addgene. Transfection was carried out with Lipofectamine 2000 (Invitrogen; Carlsbad, CA, USA).

\section{DAPI staining}

DAPI was used to determine the morphological condition of cell nuclei. Cells were fixed for fifteen minutes with 4\% paraformaldehyde, and stained with DAPI (Beyotime, Nanjing, Jiangsu, China). Cells were washed to remove the stain and results were visualized using a fluorescence microscope. Apoptosis manifested itself as fragmented or condensed cells.

\section{Flow cytometry}

Flow cytometry was used to confirm apoptosis. Cells were digested in trypsin without EDTA. Approximately $1 \times 10^{5}$ cells were resuspended in binding buffer, which included $2 \mu \mathrm{l} 50 \mu \mathrm{g} / \mathrm{ml}$ PI (propidium iodide) and $2 \mu \mathrm{l} 20 \mu \mathrm{g} / \mathrm{ml}$ annexin V-FITC. The cells was incubated for fifteen minutes in the dark. A flow cytometer (BD; San Jose, CA, USA) with $488 \mathrm{~nm}$ laser excitation was used. Following one hour of cell staining, an assessment of the cell distribution was conducted utilizing Modfit LT software (BD; San Jose, CA, USA). At least 10, 000 cells were collected for each sample with the flow rate at 250 to 300 cells/s. Cells were considered apoptotic when negative for PI and positive for annexin V-FITC. For cycle assessment, cells were digested using trypsin without EDTA, followed by washing twice in cold PBS 48 hours before transfections were performed. Seventy percent ethanol was added to fix the cells and they were maintained overnight at $4^{\circ} \mathrm{C}$. Subsequent to ethanol elimination via centrifugation, cells were washed twice in PBS. Cells were suspended in PBS and incubated in RNase $(10 \mu \mathrm{g} / \mathrm{ml})$ for thirty minutes at $37^{\circ} \mathrm{C}$. Subsequent to PI staining, the BD FACSCalibur was used to evaluate the cell cycle. CellQuest (BD, Franklin Lakes, NJ, USA).

\section{CCK-8 assay}

The cell counting kit-8 (CCK-8) assay was performed to determine the effect of the miR-34a mimic or inhibitor transfection on chondrocyte or Hs 819.T cell proliferation. CCK-8 solution (10 $\mu$ l) was added into every well and incubated for twelve hours. Absorbance was detected at $450 \mathrm{~nm}$ by a plate reader.

$\beta$-galactosidase staining

Senescence-associated function was measured using the $\beta$-galactosidase (SA- $\beta$-gal) staining kit (Beyotime,Nangjing, Jiangsu, China), where senescent chondrocytes stained blue. 


\section{Cellular Physiology Cell Physiol Biochem 2018;48:1304-1316 \\ \begin{tabular}{l|l} 
and Biochemistry & $\begin{array}{l}\text { DOI: 10.1159/000492090 } \\
\text { Publisned onmne: } 2018 \text { The Author(s). Published by S. Karger AG, Basel }\end{array}$ \\
\hline www.karger.com/cpb
\end{tabular} \\ Zhang et al.: MiR-34a Modulates Osteoarthritis}

\section{Wound healing assay}

In order to explore the ability of those cells to recover from injury, a wound-healing assay was conducted. Cells were grown to form a confluent monolayer in 6-well plates. Using a 200-microliter sterile pipette tip, a scratch was introduced to the confluent cells, simulating a wound. Cells were allowed to continue growing for 24 hours in the medium without serum. The microscope was used to evaluate and measure the scratchinduced wounds, whose width was assessed with ImageJ software (NIH, USA). Results were recorded using the proportion of wound closure, with the primitive width of the scratch at $100 \%$.

\section{Luciferase reporter gene assay}

Mutant as well as wild-type 3'-UTR (untranslated region) sequences of DLL1 were generated (GenePharma, Shanghai, China) and subcloned into the promoter vector (Promega; Madison, WI, USA). After recombination, plasmids were called pGL3-DLL1 3'-UTR-WT and pGL3-DLL1 3'-UTR-MUT. Cells were seeded in 24-well plates and after reaching 70\% confluence, cotransfections of additional plasmids were performed (200 ng), as well as cotransfection with a miR-34a mimic using Lipofectamine 2000 (Invitrogen; Carlsbad, CA, USA). pGL3 promoter vectors were used as controls. For luciferase normalization, cotransfections of the Renilla luciferase control reporter vector, pRL-SV40 (Promega; Madison, WI, USA), were performed in either HEK293T or Hs 819.T cells. Each experiment was conducted a minimum of 3 times.

\section{Surgical stimulation of $O A$}

Ten week old Sprague Dawley male rats (obtained from Shanghai SLAC Animal Co., Shanghai, China) were randomly assigned to 3 groups. Groups include the following: sham-operated control (Sham), OA/ $\mathrm{NC}$ (negative control), and $\mathrm{OA}$ /antagomir (miR-34a antagomir supplement). Rats in the OA/antagomir and $\mathrm{OA} / \mathrm{NC}$ groups underwent injection of either miR-34a antagomir or miR-34a negative control into the articulation six hours prior to surgery. Nine rats were randomly sacrificed at two days, four days, three weeks, four weeks, and six weeks subsequent to the surgery. The OA model was generated via surgery, according to a previous publication [25]. In short, the ligament cruciatum genu anterius or posterius, the meniscus medialis, and the ligamentum mediale of the posterior rat limbs were eliminated in order to bring about articular deformity, which led to the enhancement of damage to the articular face, as well as deterioration of joint cartilage. Six weeks after the OA model operation, sham surgery was conducted by revealing cartilage surfaces. The complete knee articulation was obtained to conduct a further evaluation. All animal experiments were approved by the Committee of Animal Experiments of Shanghai Jiao Tong University Affiliated Sixth People's Hospital.

\section{Safranin 0 staining}

The complete articulation of the knees was maintained in poly formaldehyde (4\%) and was fixed for twenty-four hours. Subsequent to traditional paraffin embedding, the articulation was cut into pieces that were five-micrometer thick. The Safranin O kit (ICH World, Woodstock, NY, USA) was used for staining. For histomorphometric assessment, area and depth of cartilage positive for safranin 0 were evaluated by Image-Pro (Media Cybernetics, MD, USA). OA scores from the stained slides were determined using a modified Mankin's scoring system.

\section{Real-time RT-PCR}

TRIZOL reagent (Invitrogen, Carlsbad, CA, USA) was utilized in order to obtain total RNA from flashfrozen tissues and cells. Equivalent quantities of RNA underwent reverse transcription in order to generate cDNA with the help of random hexamers, as well as the TaqMan miRNA reverse transcription kit (Applied Biosystems, Foster City, CA, USA) [26]. SYBR Green Real time PCR Master Mix (Toyobo, Osaka, Japan) was used for quantitative real-time PCR. Transcription of either U6 (for miRNAs) or $\beta$-actin (for mRNAs) served as the internal reference. Relative gene expression for genes of interest was evaluated using the $2^{-\Delta \Delta c t}$ method.

\section{Western blotting}

Liquid nitrogen was employed to maintain cartilage samples. Cold PBS was utilized to wash the cells. RIPA buffer was used to lyse the cells. The protein content was determined by BCA assay kit (Applygen, Beijing, China). Every specimen was supplemented with a combination of chondroitinase ABC, keratinase, and keratinase II (Sigma, St. Louis, MO, USA) without proteinase in order to eliminate GAG prior to 
electrophoretic isolation. Equivalent quantities of total protein were separated via SDS-PAGE on 10\% gels prior to transfer to PVDF membranes (Thermo Fisher Scientific, Waltham, MA, USA). After blocking with $5 \%$ milk, primary antibodies were added and incubated overnight $\left(4^{\circ} \mathrm{C}\right)$. Subsequent to washing trice, onehour incubation was performed in secondary antibody conjugated to HRP. ECL kits (Amersham, Piscataway, NJ, USA) were used to visualize protein bands. To evaluate the optical density, with $\beta$-actin serving as the internal reference, ImageJ software was utilized. Primary antibodies were as follows: DLL1, cleaved caspase 3, Bcl-2, $\beta$-actin, Bax, (Cell signaling technology, Danvers, MA, USA), p16, p-AKT, AKT (Abcam, Cambridge, MA, USA), ADAMTS-5, ADAMTS-7, MMP-1, MMP-13, thrombospondin 5 (COMP fragment), COL X, COL II, and aggrecan (Santa Cruz Biotechnology, Dallas, TX, USA).

\section{ELISA}

COL II, COL X, MMP-1, MMP-13 protein levels were evaluated in the media generated from Hs 819.T, as well cells as chondrocytes using the following ELISA kits: MMP-13 kit (Cusabio Biotech, Wuhan, China), COL $\mathrm{X}$ kit (Abbexa Ltd, Cambridge, UK), Collagen II kit (LifeSpan BioSciences, Seattle, WA, USA), Human MMP-13 kit (Abcam, Cambridge, MA, USA), Human COL X kit (LifeSpan BioSciences, Seattle, USA), Human COL II kit (LifeSpan BioSciences, Seattle, WA, USA) and Human MMP-1 kit (Abcam, Cambridge, MA, USA).

\section{Statistical analysis}

SPSS13.0 software (SPSS, IL, USA) was used to analyze data. Results were reported as means \pm SD. The Student's $t$-test was applied to evaluate the statistically significant difference between groups. Results were regarded as significant with $P<0.05$. Every procedure was conducted in triplicate.

\section{Results}

\section{MiR-34a promotes apoptosis and senescence of rat primary chondrocytes and Hs}

\section{T chondrosarcoma cells}

In order to demonstrate that miR-34a participated in OA pathogenesis, we investigated miR-34a expression in $\mathrm{OA}$ cartilages. The findings revealed that miR-34a expression was remarkably stronger in OA patients than in OA-free participants (Fig. 1A), indicating that miR-34a could participate in OA pathogenesis.

Evidence suggests that $\mathrm{OA}$ is linked to the death of chondrocytes. Consequently, the effect of miR-34a on senescence and apoptosis was explored in Hs 819.T cells, as well as primary chondrocytes. Primary chondrocytes and Hs 819.T cells were transfected with a miR-34a inhibitor or mimic and apoptosis was analyzed by western blotting, flow cytometry, and DAPI staining. SA- $\beta$-gal staining and p16, which served as a senescence marker, were used to analyze senescence. Because of limited apoptosis under the current conditions, the counteracting effects of the miR-34a inhibitor against apoptosis are difficult to evaluate. In order to understand the counteracting effects of the miR-34a inhibitor on cell death, apoptosis was triggered via TRAIL followed by transfection with a miR-34a inhibitor. As seen in Fig. 1B, the quantity of apoptotic cells in the mimic treated groups was remarkably higher than that of the negative control (NC) group. Moreover, flow cytometry results showed that transfection with the miR-34a inhibitor significantly inhibited TRAIL-induced apoptosis in both Hs 819.T chondrosarcoma cells and primary chondrocytes. The proportion of early and terminal stage apoptotic cells in the mimic treated group was remarkably higher than the NC. Compared to the TRAIL alone treatment group, the inhibitor of miR-34a suppressed the number of apoptotic cells (Fig. 1C). Surprisingly, the miR-34a mimic transfection enhanced translation of cleaved caspase- 3 and the ratio of Bcl-2 to Bax proteins in Hs 819.T cells, primary chondrocytes and HC. The miR-34a inhibitor significantly inhibited TRAIL-mediated caspase 3 activation (Fig. 1D). Cells characterized by positive SA- $\beta$-gal were enhanced in chondrocytes that underwent transfection with the miR-34a mimic (Fig. 1E). Similar to SA$\beta$-gal staining, protein levels of p16 were enhanced in chondrocytes, which were transfected with the miR-34a mimic (Fig. 1F). 
Fig. 1. miR-34a promoted apoptosis and senescence of rat chondrocytes and Hs 819.T cells. (A) Relative miR-34a levels in clinical tissues were determined by qRT-PCR. (B) Photomicrographs of the cells stained with DAPI. (C) Cell apoptosis of chondrocytes and chondrosarcoma cells were analyzed by flow cytometry. (D) Protein levels of cleaved caspase-3, Bax, and Bcl-2 were measured by western blotting. (E) SA- $\beta$-gal stained positive cells were identified in chondrocytes following transfections. (F) The expression levels of p16 were determined by western blotting. Results in (C) and (E) were presented as means \pm SD $(n=3)$. ***, $\mathrm{P}<0.001$; **, $\mathrm{P}<0.01$.

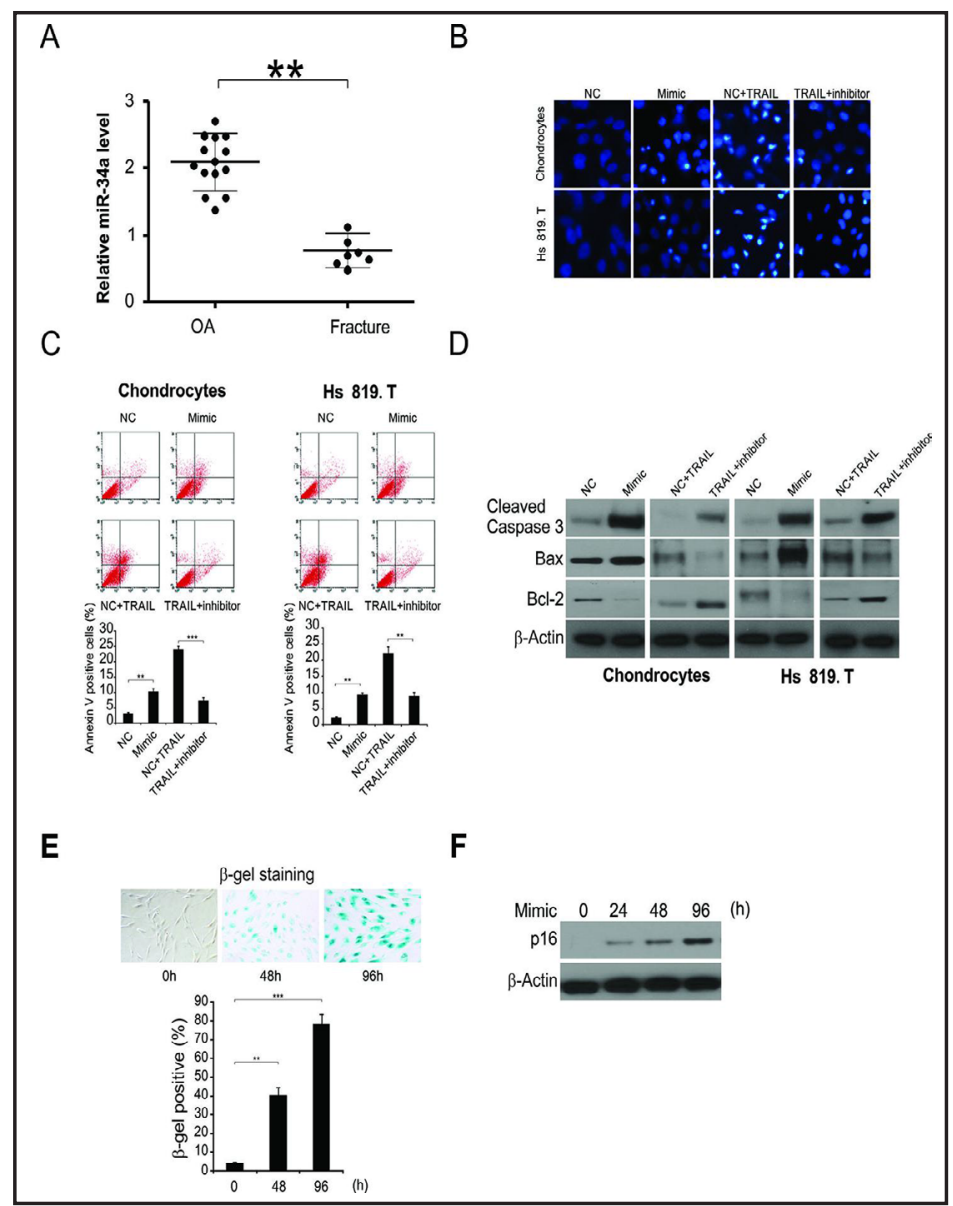

Fig. 2. miR-34a suppressed the growth and migration of rat chondrocytes and Hs 819.T cells. (A) miR-34a mimic and inhibitor altered the proliferation of $\mathrm{HC}$, chondrocytes and Hs 819.T cells. (B) The miR-34a mimic hindered the closure of a scratch-induced wound, while the inhibitor promoted the scratch induced wound healing. Results in (B) were presented as means \pm SD $(n=3) . * *$, $\mathrm{P}<0.01 ; *, \mathrm{P}<0.05$.

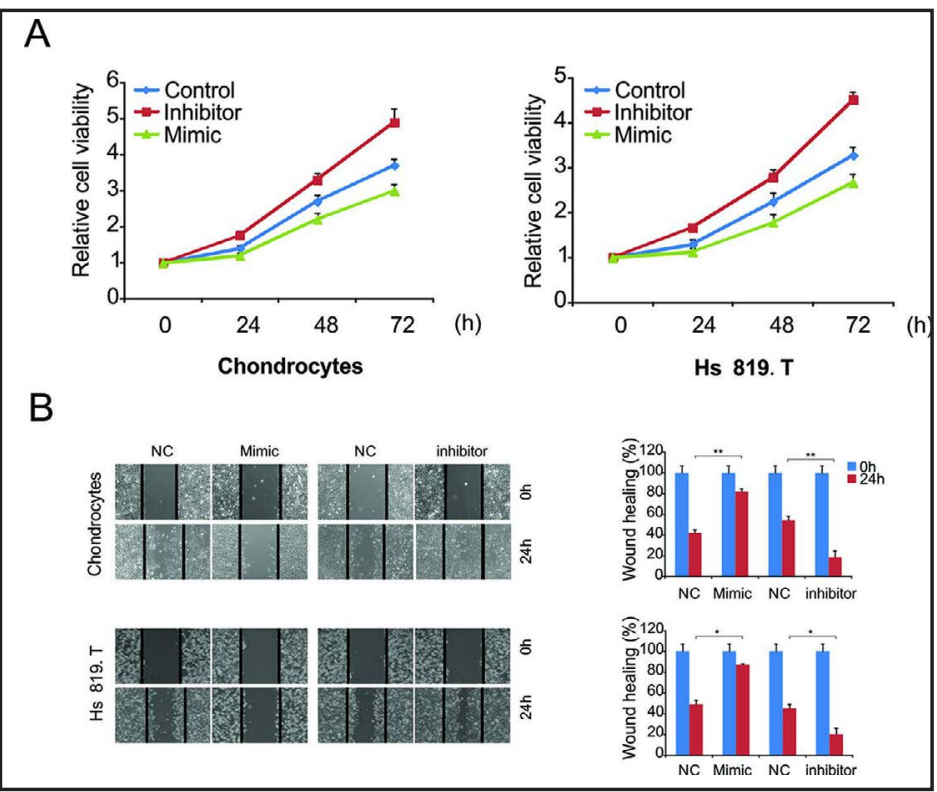

MiR-34a inhibited the growth of rat chondrocytes and Hs 819.T chondrosarcoma cells, and suppressed scratch wound closure

Enhancing proliferation as well as differentiation of chondrocytes via modulation of gene expression could be useful for OA treatment. Consequently, the effect of miR-34a 
on cell growth was assessed in $\mathrm{HC}$, Hs 819.T chondrosarcoma cells and primary chondrocytes using the CCK-8 assay. The miR-34a mimic transfection remarkably suppressed the growth of these cells (Fig. 2A). However, transfection with miR34a inhibitor significantly enhanced the growth of cells above. Additionally, wound healing assay was conducted in order to explore the influence of miR-34a on the ability to recover from injury. The findings above revealed that the miR-34a mimic reduced the recovery from scratch-induced damage, while the miR-34a inhibitor enhanced the recovery (Fig. 2B). In brief, miR-34a inhibited the growth of Hs 819.T chondrosarcoma cells as well as primary chondrocytes. Additionally, miR-34a suppressed the closure of scratch wounds, suggesting that elimination of miR-34a may lead to enhanced OA cartilage recovery.

MiR-34a altered the secretion of molecules associated with cartilage degeneration

The unregulated production of extracellular matrix (ECM), and the degeneration of joint chondrocytes, is essential contributors to cartilage deterioration. MMPs serve as crucial proteins in hydrolysis, leading to ECM degeneration. MMP-13 and MMP-1 are able to directly degrade COL II, the most prevalent collagen in the ECM. Hypertrophic chondrocytes are utilized to produce COL $\mathrm{X}$, which is able to serve as a marker of advanced OA. Transfection with the miR-34a mimic remarkably enhanced the generation of COL X, MMP-13, and MMP-1 in the HC,

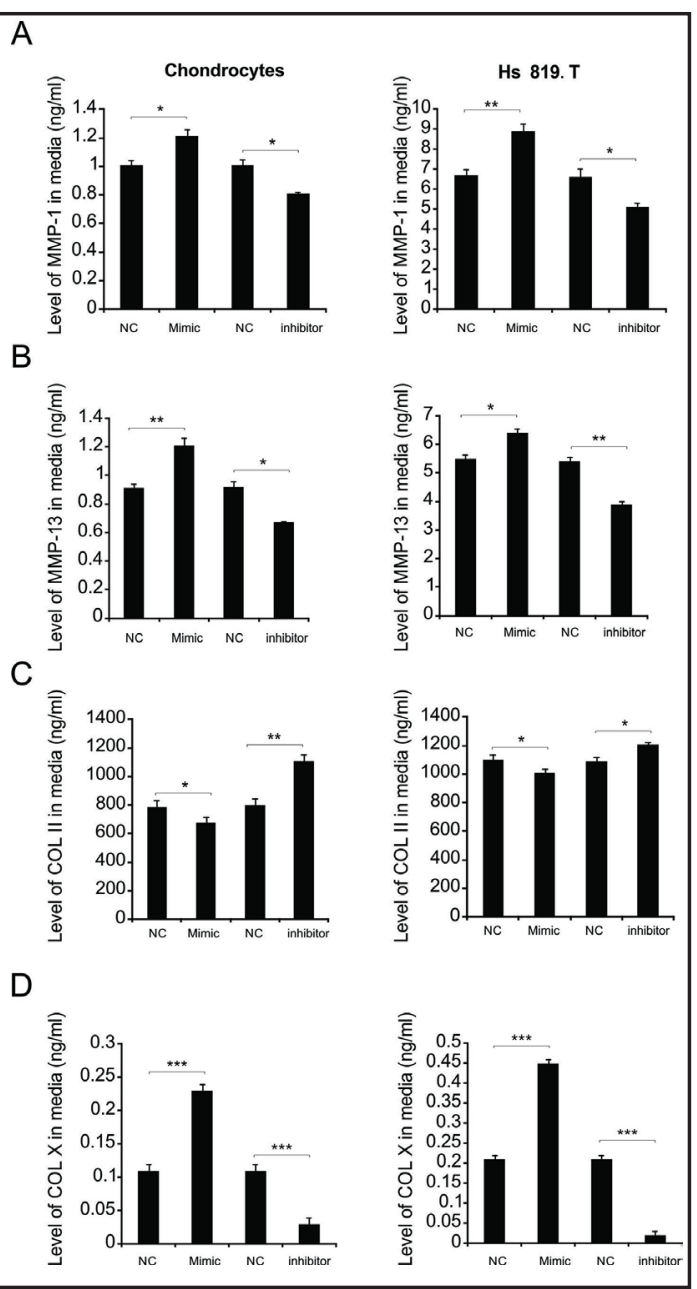

Fig. 3. miR-34a regulated cartilaginous degenerationrelated molecules. (A-D) Concentrations of MMP-1, MMP-13, COL II, and COL X in HC, rat chondrocytes and Hs 819.T cells culture media after transfection with miR-34a mimic or inhibitor were determined by ELISA assay. Results in (B) were presented as means $\pm \mathrm{SD}(\mathrm{n}=3)$. $^{* *}, \mathrm{P}<0.001$; $^{* *}, \mathrm{P}<0.01 ;{ }^{*}, \mathrm{P}<0.05$. Hs 819.T cells and primary chondrocytes.

However, it decreased transcription and secretion of COL II. Transfection with the miR-34a inhibitor had adverse effects on the generation and secretion of COL X, COL II, MMP-1, and MMP-13 (Fig. 3A-3D).

\section{Negative modulation of DLL1 via miR-34a in Hs 819.T chondrosarcoma cells and rat primary chondrocytes.}

A bioinformatics evaluation was conducted in search of possible target genes of miR34a. As a result, DLL1 was found to be a potential target. In order to explore the interaction between miR-34a and the DLL1 3'-UTR, the miR-34a binding region was mutated in the DLL1 3'-UTR (Fig. 4A). Subsequent to luciferase vector co-transfection with DLL1 3'-UTR as well as the miR-34a mimic, the miR-34a mimic was discovered to be unable to inhibit the expression of luciferase (Fig. 4B), suggesting that the interrelation between miR-34a and the binding site of the DLL1 3'-UTR directly modulates the expression of the luciferase reporter. 
Fig. 4. miR-34a direct targeted $3^{\prime}$ UTR of DLL1. (A) Schematic representation of miR-34a predicted binding site in the $3^{\prime}$ UTR of DLL1 mRNAs. (B) The DLL1 3'-UTR reporter vector was transfected into $\mathrm{NC}$ or miR-34a mimic transfected Hs 819.T human chondrosarcoma cells and the reporter activities were compared. (C) The cells were treated as indicated, the mRNA level of DLL1 was analyzed by RT-PCR. (D) The cells were treated as indicated, the protein level of DLL1 was analyzed

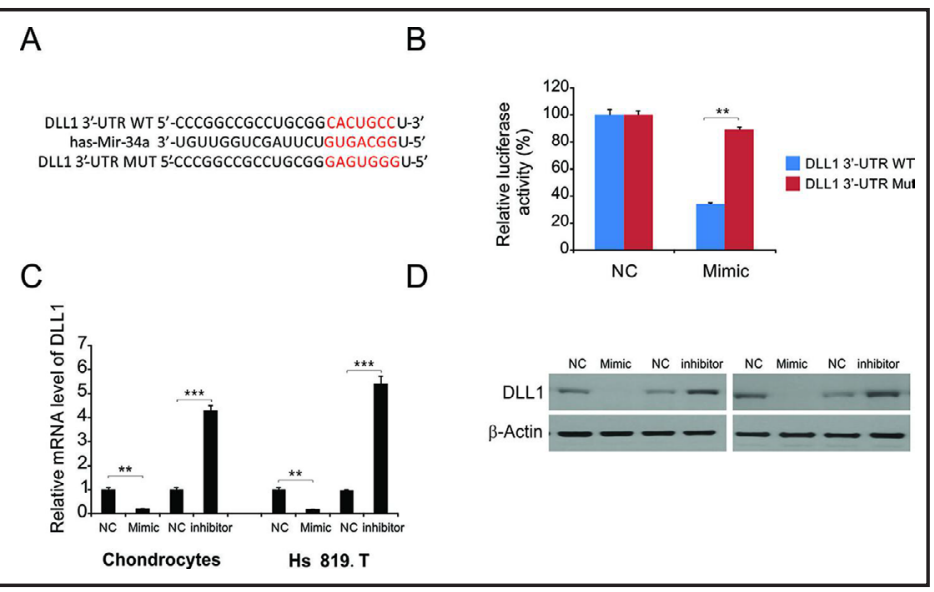
by western blotting. Results in (B) and (C) were presented as means $\pm S D(n=3)$. ${ }^{* *}, P<0.01$.

Hs 819.T chondrosarcoma cells and primary chondrocytes were transfected with a miR-34a inhibitor or mimic. The effect of miR-34a on secretion and generation of DLL1 was evaluated. The findings indicated that transcription, secretion, and translation of DLL1 were reversely modulated through miR-34a (Fig. 4C and 4D).

\section{DLL1 was essential to cell death triggered by an miR-34a mimic and lead to reduced}

AKT levels in chondrocytes

Western blotting was utilized to determine the participation of miR-34a in the modulation of the PI3K/AKT pathway. The findings revealed that the level of total AKT and p-AKT declined in the groups that underwent miR-34a transfection (Fig. 5A). Moreover, it was discovered that excessive DLL1 expression caused the up-regulation of p-AKT and AKT through miR-34a (Fig. 5B).

Because miR-34a was able to inhibit DLL1 generation, our research hypothesized that the influence of the miR-34a mimic on death, growth, and migration of Hs 819.T chondrosarcoma cells and primary chondrocytes was caused by a lack of DLL1. In order to prove this hypothesis, Hs 819.T cells and primary chondrocytes were transfected with the miR-34a mimic, excessively expressing DLL1. Subsequent to the 48 hours of treatment, cell growth and apoptosis were analyzed and the ability to recover from injury was also detected. The miR-34a mimic caused excessive DLL1 expression, decreasing the level of cleaved caspase-3 and triggering a decline in cell death (Fig. 5C-5D). Excessive DLL1 expression reduced the growth suppression via the mimic (Fig. 5E) and stimulated wound closure in comparison to cells merely supplemented with the miR-34a mimic (Fig. 5F). This indicates that miR-34a modulated chondrosarcoma activities via DLL1.

\section{MiR-34a antagomir decreased death of joint chondrocytes and hindered the loss of}

knee articular cartilage in rats with surgery-induced $O A$

According to the outcome of our in vitro research, we hypothesized that miR-34a elimination could reduce the death of OA chondrocytes in vivo. In order to verify this theory, rats received miR-34a antagomir before OA stimulation and apoptosis was measured six weeks after surgical OA induction. The specificity of antagomir for miR-34a was examined in vitro. In the $\mathrm{OA} / \mathrm{NC}$ group, the concentration of miR-34a quickly reached its peak two days after surgical induction of $\mathrm{OA}$ and declined after that (Fig. 6A). Injection of miR-34a antagomir into the joints remarkably inhibited miR-34a upregulation triggered by OA (Fig. 6A). In the $\mathrm{OA} / \mathrm{NC}$ group, the concentration of DLL1 in the cartilage was higher in comparison to the Sham group at 2 days, four weeks, and six weeks following surgery. DLL1 was noticeably enhanced via preliminary supplementation of miR-34a antagomir (Fig. 6B). Apoptosis was 
Fig. 5. DLL1 affected miR-34a mimic mediated AKT inhibition and apoptosis. (A) Hs 819.T cells were treated with miR-34a mimic and protein levels were determined by western blotting. (B) Hs 819.T cells transfected with DLL1 siRNA were treated with miR-34a mimic, protein levels were determined by western blotting. (C) The protein level of cleaved caspase-3, Bax, and $\mathrm{Bcl}-2$ were determined by western blotting. (D) Percentage of apoptotic cells determined by flow cytometry after treatment with miR-34a mimic or a combination of DLL1 overexpression and the mimic. (E) Cell proliferation was determined by the CCK- 8 kit. (F) Percentage of wound closure was determined by scratch wound assay. Results in (D), (E), and (F) were presented as means \pm SD $(\mathrm{n}=3)$. **, $\mathrm{P}<0.01$; $^{*}, \mathrm{P}<0.05$.

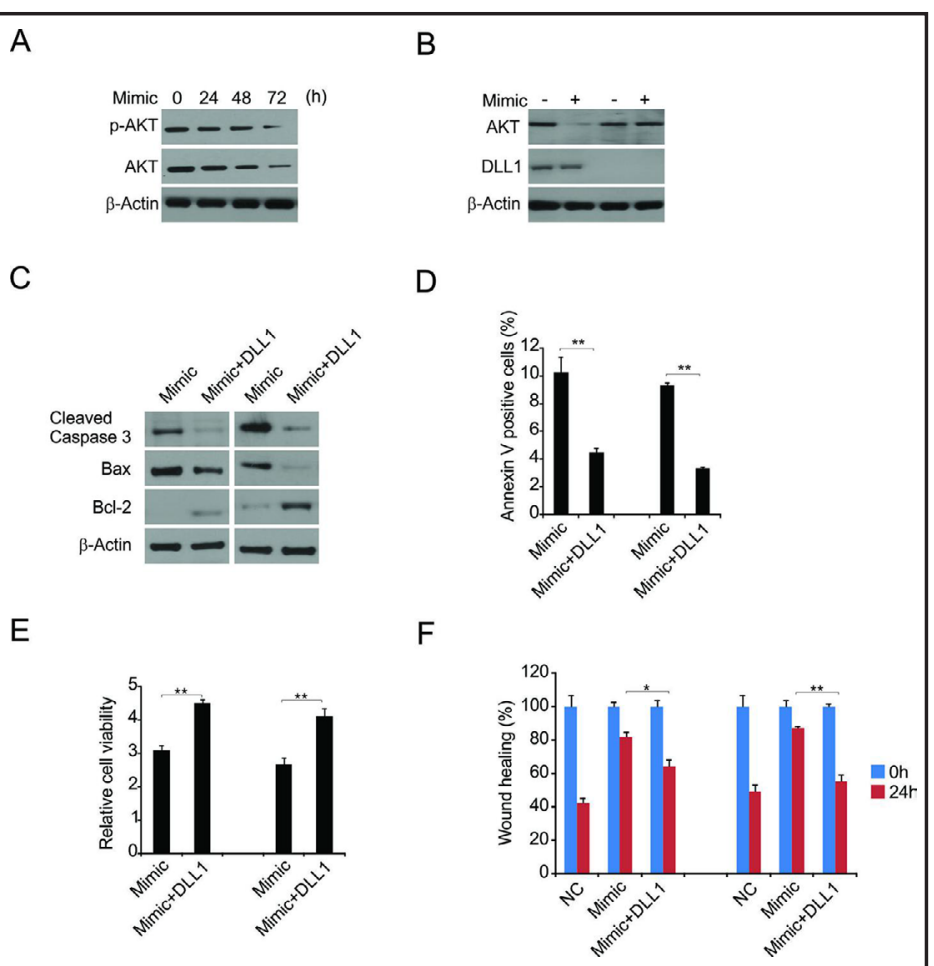

evaluated by determining the concentration of cleaved caspase-3, which was significantly higher after OA stimulation. Preliminary supplementation of miR-34a antagomir was able to reduce apoptosis (Fig. 6C). OA is characterized by a disequilibrium between generation and degeneration of ECM, which brings about a decrease of cartilage matrix. Cartilage covering articular surfaces is able to be thoroughly depleted. Next, we examined miR-34a antagomir's influence on the loss of cartilage. After OA stimulation, translation of ADAMTS-7, ADAMTS-5, MMP-13, and MMP-1 was promoted, leading to cartilage loss through degradation of COL II, aggrecan, and COMP. The miR-34a antagomir was able to suppress the generation of matrix degrading enzymes and decrease the degeneration of COL II, aggrecan, and COMP (Fig. 6D). Safranin 0/fast green staining was utilized in order to determine the effect of miR-34a antagomir on the loss of cartilage. Rats in the Sham group displayed the entire structure of the Articulatio genus with uniform safranin 0 staining (Fig. 6E). Nevertheless, OA/NC group mice displayed the clinical manifestations of $\mathrm{OA}$, such as the generation of superficial fissures, decreased safranin $O$ staining on superficial as well as polymorphic layers, and occurrence of large chondrocytes in deep layers (Fig. 6E). A histomorphometric assessment revealed that cartilage depth of the Articulatio genus was significantly reduced in OA/NC rats (Fig. $6 \mathrm{~F}$ ). Additionally, Mankin's score of $\mathrm{OA} / \mathrm{NC}$ rats was significantly elevated in comparison to Sham rats (Fig. 6G). However, preliminary supplementation with miR-34a antagomir was able to remarkably suppress cartilage loss as well as lower the Mankin's score, proving that degradation of the articular genus cartilage was remarkably suppressed in OA rats via the miR-34a antagomir.

\section{Discussion}

OA features chronic degradation of joint cartilage, subchondral bone sclerosis, and degradation of the articular fringe [6]. OA is prevalent on the articular surfaces of the hands, elbows, hips, and knees [27]. Joint cartilage, ligaments, subchondral bone, capsule, and adjacent muscles are vulnerable to $\mathrm{OA}[28,29]$. Currently, it is widely accepted that $\mathrm{OA}$ is 
Fig. 6. miR-34a antagomir decreased apoptosis of articular chondrocytes and impeded the loss of cartilage in the knee joint of surgically induced rat osteoarthritic model. (A) After surgery, miR-34a level in the tibial cartilage was determined by qRTPCR. (B) After surgery, DLL1 in the tibial cartilage was determined by western blotting. (C) Cleavage of caspase- 3 in the cartilage was determined by western blotting. (D) Protein levels of ADAMTS-5, -7, MMP-1, MMP-13, COMP fragment, COL II, aggrecan, and COL X were determined by western blotting. (E) Representative safranin 0 staining of the knee joint. (F) Cartilage thickness in the tibia was determined by histomorphometry.

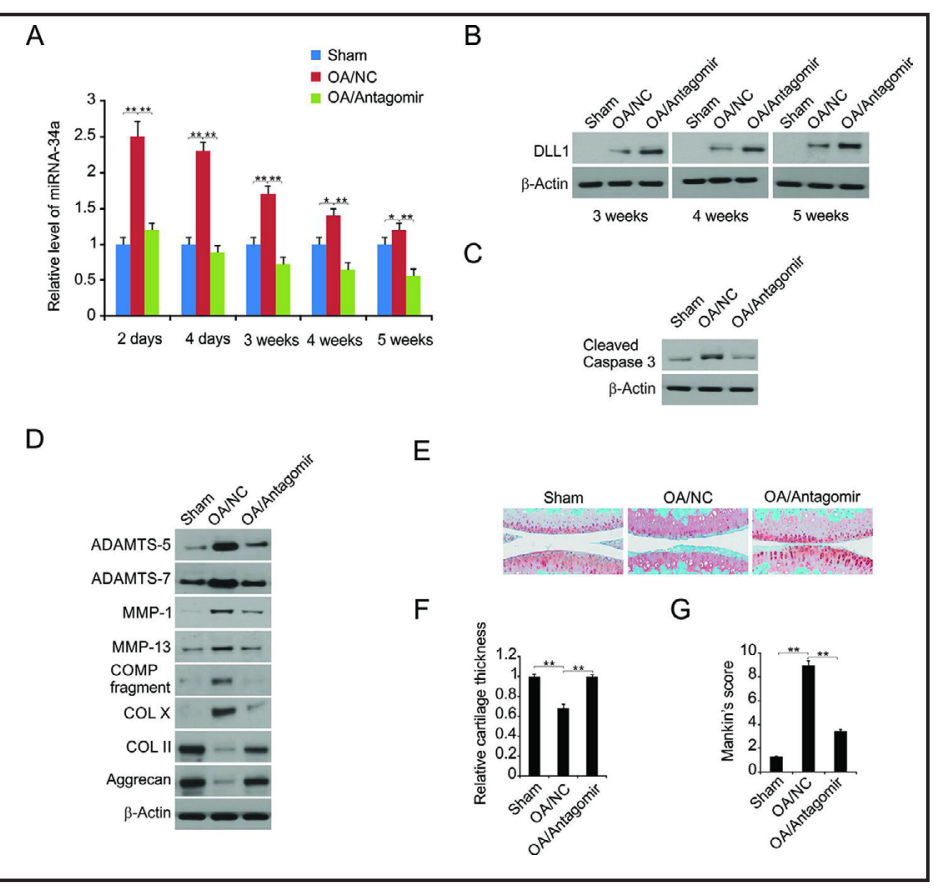

(G) Degenerative changes were analyzed by Mankin's scoring. Results in $(A),(F)$ and $(G)$ were presented as means \pm SD (n=3). **, $P<0.01$, $*, \mathrm{P}<0.05$.

brought about via multiple factors, such as biomechanical forces, genetic heritage, metabolic distress, chondrocyte death, cytokine presence, cartilage health, and the presence of free radicals [30-32].

The miRNA family consists of single chain, non-coding small RNA molecules that are usually twenty-two to twenty-three nucleotides in length $[33,34]$. The miRNA is prevalent in animals, plants, and microbiota. In recent years, considerable proof revealed that multiple miRNAs participate in the generation and progression of OA [35]. Multiple studies proved that miR-22 directly suppressed PRARA, as well as BMP-7 expression in OA, causing elevated IL-1 and MMP-13 expression and leading to the degradation of chondrocytes and matrix $[36,37]$. Furthermore, it was demonstrated that several other miRNAs participated in OA progression, including miR-130 and miR-483 [38].

To determine the role of miR-34a in $\mathrm{OA}$, we initially examined the in vitro influence of knock-down or excessive expression of miR-34a on growth, death, and scratch wound recovery of rat primary chondrocytes, as well as human chondrosarcoma cells via transfection with miR-34a inhibitors or mimics. Following transfection verification via qRTPCR, we discovered that elevated miR-34a expression remarkably enhanced cell death and suppressed the proliferation of cells. However, miR-34a downregulation led to the noticeable suppression of cell death and increase in cell proliferation. Moreover, the outcome of wound healing assays showed that the miR-34a mimic transfection prevented recovery of scratchinduced cell damage. Despite the fact that is direct proof showing that miR-34a can enhance chondrocyte death is lacking, the trigger of miR-34 on cell death has been reported in a variety of malignant cells, as well as several normal cell types, such as glioblastoma cells and neurons. In our research, we discovered that a miR-34a mimic was able to enhance the stimulation of caspase-3, which is known to participate in cell death.

The correlation between chondrocyte death and the generation and progression of OA is still controversial. Death of chondrocytes usually takes place in the terminal stages of OA progression. During this stage, the number of cells in highly fibrillated cartilage matrix is significantly decreased, indicating that the death of chondrocyte serves as a terminal event in OA $[39,40]$. Nevertheless, several studies claimed that chondrocyte death served as an 
initiating event $[41,42]$. In spite of this, increasingly more researchers have suggested that the death of chondrocyte participates in cartilage degradation in OA [43, 44]. Consequently, chondrocyte death appears to be a promising treatment target in OA[44]. Risk factors leading to chondrocyte death in $\mathrm{OA}$ are diverse, including nitrous oxide, staurosporine, Fas ligand, hypertrophy, and a shortage of ECM or adhesion [43]. Our research proved that in vivo cartilage loss brought about by chondrocyte death was remarkably suppressed via knockdown of miR-34a using a miR-34a antagomir. In addition, it was discovered that excessive miR-34a expression triggered the expression of ECM degrading enzymes, including MMP-13 and MMP-1 in rat primary chondrocytes as well as human Hs 819.T cells. However, miR34a downregulation remarkably inhibited generation of MMP-13, MMP-1, ADAMTS-7, and ADAMTS-5 in vivo with increasing concentrations of ECM components, including COL II, COL $\mathrm{X}, \mathrm{COMP}$, and aggrecan.

Noticeably, our research proved that miR-34a is able to directly modulate the expression of DLL1. We determined a binding region of miR-34a in the 3'-UTR of DLL1 mRNA. The miR-34a mimic significantly suppressed the expression of luciferase via DLL1, which was eliminated via mutations in the 3'-UTR of DLL1. Furthermore, the miR-34a mimic was able to remarkably suppress the expression of DLL1. The miR-34a inhibitor enhanced expression of DLL1. Consequently, we hypothesized that miR-34a could trigger chondrocyte death via DLL1, which was verified when DLL1 expression eliminated the influence of the miR-34a mimic on cell death, proliferation, and wound recovery in chondrosarcoma cells.

Our research proved that death, as well as senescence, was triggered by miR-34a via DLL1 by modulating the AKT pathway in rat chondrocytes and Hs 819.T chondrosarcoma cells. miR-34a knock-downs and miR-34a antagomir inhibited the death of joint chondrocytes and the loss of cartilage. These findings indicate that DLL1 and miR-34a are promising targets for OA treatment.

\section{Acknowledgements}

This project was supported by the National Natural Science Foundation of China (No. 81601956).

\section{Disclosure Statement}

The authors declare to have no competing interests.

\section{References}

1 Brandt KD, Fife RS: Ageing in relation to the pathogenesis of osteoarthritis. Clin Rheum Dis 1986;12:117130 .

-2 Loeser RF, Collins JA, Diekman BO: Ageing and the pathogenesis of osteoarthritis. Nat Rev Rheumatol 2016;12:412-420.

-3 Mobasheri A: Role of chondrocyte death and hypocellularity in ageing human articular cartilage and the pathogenesis of osteoarthritis. Med Hypotheses 2002;58:193-197.

4 Buraimoh MA, Massie LW, Montgomery DM: Lateral Atlantoaxial Osteoarthritis: A Narrative Literature Review. Clin Spine Surg 2017;30:433-438.

-5 Rayegani SM, Raeissadat SA, Heidari S, Moradi-Joo M: Safety and Effectiveness of Low-Level Laser Therapy in Patients With Knee Osteoarthritis: A Systematic Review and Meta-analysis. J Lasers Med Sci 2017;8:S12-S19.

6 Block TJ, Garza JR: Regenerative Cells for the Management of Osteoarthritis and Joint Disorders: A Concise Literature Review. Aesthet Surg J 2017;37:S9-S15. 


\section{Cellular Physiology Cell Physiol Biochem 2018;48:1304-1316

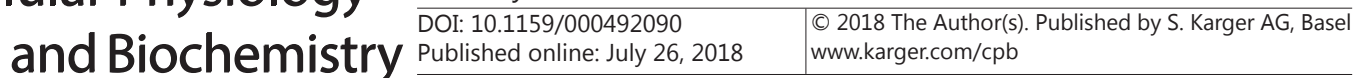 \\ Zhang et al.: MiR-34a Modulates Osteoarthritis}

7 Miyaki S, Lotz MK: Extracellular vesicles in cartilage homeostasis and osteoarthritis. Curr Opin Rheumatol 2018;30:129-135.

8 Rahmati M, Nalesso G, Mobasheri A, Mozafari M: Aging and osteoarthritis: Central role of the extracellular matrix. Ageing Res Rev 2017;40:20-30.

-9 Kalaitzoglou E, Griffin TM, Humphrey MB: Innate Immune Responses and Osteoarthritis. Curr Rheumatol Rep 2017;19:45.

10 Nguyen LT, Sharma AR, Chakraborty C, Saibaba B, Ahn ME, Lee SS: Review of Prospects of Biological Fluid Biomarkers in Osteoarthritis. Int J Mol Sci 2017;18:

11 He L, Hannon GJ: MicroRNAs: small RNAs with a big role in gene regulation. Nat Rev Genet 2004;5:522-531.

12 Yu C, Chen WP, Wang XH: MicroRNA in osteoarthritis. J Int Med Res 2011;39:1-9.

13 Zhang M, Lygrisse K, Wang J: Role of MicroRNA in Osteoarthritis. J Arthritis 2017;6:239.

14 Tornero-Esteban P, Rodriguez-Rodriguez L, Abasolo L, Tome M, Lopez-Romero P, Herranz E, Gonzalez MA, Marco F, Moro E, Fernandez-Gutierrez B, Lamas JR: Signature of microRNA expression during osteogenic differentiation of bone marrow MSCs reveals a putative role of miR-335-5p in osteoarthritis. BMC Musculoskelet Disord 2015;16:182.

15 Li XJ, Ren ZJ, Tang JH: MicroRNA-34a: a potential therapeutic target in human cancer. Cell Death Dis 2014;5:e1327.

16 Rokavec M, Li H, Jiang L, Hermeking H: The p53/miR-34 axis in development and disease. J Mol Cell Biol 2014;6:214-230.

17 Wang R, Ma J, Wu Q Xia J, Miele L, Sarkar FH, Wang Z: Functional role of miR-34 family in human cancer. Curr Drug Targets 2013;14:1185-1191.

18 Ding J, Li M, Wan X, Jin X, Chen S, Yu C, Li Y: Effect of miR-34a in regulating steatosis by targeting PPARalpha expression in nonalcoholic fatty liver disease. Sci Rep 2015;5:13729.

19 Fiúza UM, Arias AM: Cell and molecular biology of Notch. J Endocrinol 2007;194:459-474.

20 Khait I, Orsher Y, Golan O, Binshtok U, Gordon-Bar N, Amir-Zilberstein L, Sprinzak D: Quantitative analysis of Delta-like 1 membrane dynamics elucidates the role of contact geometry on Notch signaling. Cell Rep 2016;14:225-233.

21 Jason S, Cui W: Proliferation, survival and metabolism: the role of PI3K/AKT/mTOR signalling in pluripotency and cell fate determination. Development 2016;143:3050-3060.

2222 Guo LY, Li YM, Qiao L, Liu T, Du YY, Zhang JQ He WT, Zhao YX, He DQ: Notch2 regulates matrix metallopeptidase 9 via PI3K/AKT signaling in human gastric carcinoma cell MKN-45. World J Gastroenterol 2012;18:7262.

23 Cornejo MG, Mabialah V, Sykes SM, Khandan T, Celso CL, Lopez CK, Rivera-Muñoz P, Rameau P, Tothova Z, Aster JC: Crosstalk between NOTCH and AKT signaling during murine megakaryocyte lineage specification. Blood 2011;118:1264-1273.

24 Sangphech N, Osborne BA, Palaga T: Notch signaling regulates the phosphorylation of Akt and survival of lipopolysaccharide-activated macrophages via regulator of G protein signaling 19 (RGS19). Immunobiology 2014;219:653-660.

-25 Lorenz J, Grassel S: Experimental osteoarthritis models in mice. Methods Mol Biol 2014;1194:401-419.

- 26 Tong J, Tan S, Zou F, Yu J, Zhang L: FBW7 mutations mediate resistance of colorectal cancer to targeted therapies by blocking Mcl-1 degradation. Oncogene 2017;36:787-796.

27 Wood AM, Brock TM, Heil K, Holmes R, Weusten A: A Review on the Management of Hip and Knee Osteoarthritis. Int J Chronic Dis 2013;2013:845015.

28 Blum MA, Ibrahim SA: Race/ethnicity and use of elective joint replacement in the management of endstage knee/hip osteoarthritis: a review of the literature. Clin Geriatr Med 2012;28:521-532.

29 Tong J, Tan S, Nikolovska-Coleska Z, Yu J, Zou F, Zhang L: FBW7-Dependent Mcl-1 Degradation Mediates the Anticancer Effect of Hsp90 Inhibitors. Mol Cancer Ther 2017;16:1979-1988.

-30 Topol GA, Podesta LA, Reeves KD, Giraldo MM, Johnson LL, Grasso R, Jamin A, Clark T, Rabago D: Chondrogenic Effect of Intra-articular Hypertonic-Dextrose (Prolotherapy) in Severe Knee Osteoarthritis. PM R 2016;8:1072-1082.

-31 Rabago D, van Leuven L, Benes L, Fortney L, Slattengren A, Grettie J, Mundt M: Qualitative Assessment of Patients Receiving Prolotherapy for Knee Osteoarthritis in a Multimethod Study. J Altern Complement Med 2016;22:983-989. 


\section{Cellular Physiology Cell Physiol Biochem 2018;48:1304-1316

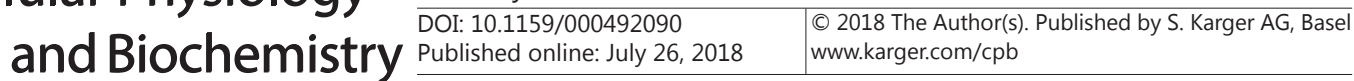 \\ Zhang et al.: MiR-34a Modulates Osteoarthritis}

-32 Sit RW, Chung V, Reeves KD, Rabago D, Chan KK, Chan DC, Wu X, Ho RS, Wong SY: Hypertonic dextrose injections (prolotherapy) in the treatment of symptomatic knee osteoarthritis: A systematic review and meta-analysis. Sci Rep 2016;6:25247.

33 Kumar B, Lupold SE: MicroRNA expression and function in prostate cancer: a review of current knowledge and opportunities for discovery. Asian J Androl 2016;18:559-567.

34 Cai Y, Yu X, Hu S, Yu J: A brief review on the mechanisms of miRNA regulation. Genomics Proteomics Bioinformatics 2009;7:147-154.

- 35 Xu B, Li YY, Ma J, Pei FX: Roles of microRNA and signaling pathway in osteoarthritis pathogenesis. J Zhejiang University-SCIENCE B 2016;17:200-208.

36 Le LT, Swingler TE, Clark IM: the role of microRNAs in osteoarthritis and chondrogenesis. Arthritis Rheumatol 2013;65:1963-1974.

37 Tong J, Wang P, Tan S, Chen D, Nikolovska-Coleska Z, Zou F, Yu J, Zhang L: Mcl-1 Degradation Is Required for Targeted Therapeutics to Eradicate Colon Cancer Cells. Cancer Res 2017;77:2512-2521.

38 Wu C, Tian B, Qu X, Liu F, Tang T, Qin A, Zhu Z, Dai K: MicroRNAs play a role in chondrogenesis and osteoarthritis. Int Mol Med 2014;34:13-23.

39 Goldring MB: Chondrogenesis, chondrocyte differentiation, and articular cartilage metabolism in health and osteoarthritis. Thera Advanc Musculoskel Dis 2012;4:269-285.

40 Van der Kraan P, Van den Berg W: Chondrocyte hypertrophy and osteoarthritis: role in initiation and progression of cartilage degeneration? Osteoarthrit Cart 2012;20:223-232.

$>41$ Loeser RF: Aging and osteoarthritis: the role of chondrocyte senescence and aging changes in the cartilage matrix. Osteoarthrit Cart 2009;17:971-979.

-42 Li Y, Wei X, Zhou J, Wei L: The age-related changes in cartilage and osteoarthritis. BioMed Res Int 2013;2013:916530.

-43 Takács-Buia L, Iordachel C, Efimov N, Caloianu M, Montreuil J, Bratosin D: Pathogenesis of osteoarthritis: chondrocyte replicative senescence or apoptosis? Cytometry Part B: Clinical Cytometry 2008;74:356-362.

44 Hwang HS, Kim HA: Chondrocyte apoptosis in the pathogenesis of osteoarthritis. Int J Mol Sci2015;16:26035-26054. 